\title{
Influencia de la lesión deportiva en los cambios del estado de ánimo y de la ansiedad precompetitiva en futbolistas
}

\author{
Influence of sports injury changes in mood and \\ precompetitive anxiety in soccer players
}

\section{Influência das alteraçóes de lesão esportes de humor e ansiedade pré-competitiva em jogadores de futebol}

\author{
Aurelio Olmedilla, Enrique Ortega y José María Gómez
}

Universidad de Murcia

Resumen: El estudio de las relaciones entre lesión y aspectos psicológicos del deportista ha aumentado en los últimos años, aunque todavía quedan muchos interrogantes por responder. El objetivo del presente estudio fue analizar la incidencia de la lesión sobre los posibles cambios en el estado de ánimo y en la ansiedad precompetitiva de los futbolistas. La muestra estuvo formada por 13 jugadores de equipos de fútbol masculino que se lesionaron durante el desarrollo de la temporada 2011-2012. El rango de edad fue de 21 a 29 años, con una media de 24.58 ańos ( $D T=3.42$ ). Las lesiones que sufrieron los jugadores fueron desde el punto de vista de la gravedad de carácter moderado. Se llevó a cabo un diseńo longitudinal, en el se tomaron los datos durante una temporada completa de competición, evaluando en cada partido oficial de competición la ansiedad competitiva, el estado de ánimo, y se registraron las lesiones producidas en entrenamiento y en partido. Los resultados indican que los futbolistas manifestaban antes de la lesión niveles superiores de tensión y de autoconfianza; y después de haber sufrido una lesión manifestaban niveles superiores de depresión, ansiedad cognitiva y ansiedad somática. Se discuten los resultados y se proponen aplicaciones prácticas derivadas de los mismos

Palabras clave: Estado de ánimo, Ansiedad precompetitiva, Lesión deportiva, Futbolistas

Abstract: The study of the relationship between injury and psychological aspects of sport has increased in recent years, although many questions remain unanswered. The aim of this study was to analyze the incidence of injury on changes in mood and precompetitive anxiety of the players. The sample consisted of 13 players for men's soccer teams who were injured during the course of the 2011-2012 season. The age range was 21 to 29 years, with a mean of 24.58 years $(S D=3.42)$. The injuries to players were from the point of view of the severity of moderate character. They conducted a longitudinal design, the data were taken during a full season of competition, evaluating each official competition match competitive anxiety, mood, and injuries in training and match recorded. The results indicate that the players before the injury manifested higher levels of stress and self-confidence, and after suffering an injury manifested higher levels of depression, cognitive anxiety and somatic anxiety. Results are discussed and practical applications derived from them are proposed.

Key words: Mood state, Precompetitive anxiety, Sport injury, Soccer players.

Resumo: $\mathrm{O}$ estudo da relação entre lesóes e aspectos psicológicos do esporte tem aumentado nos últimos anos, embora muitas perguntas continuam sem resposta. O objetivo deste estudo foi analisar a incidência de lesôes em mudanças de humor e ansiedade pré-competitiva dos jogadores. A amostra consistiu de 13 jogadores para times de futebol dos homens que foram feridos durante o decorrer da temporada 2011-2012. A faixa etária era de 21 a 29 anos, com uma média de 24,58 anos $(\mathrm{DP}=3,42)$. As lesóes de jogadores foram a partir do ponto de vista da gravidade de carácter moderado. Foi realizado a projeto longitudinal, os dados foram tiradas durante uma temporada completa da concorrência, avaliando cada ansiedade árbitro competição competitivo, humor e lesóes em treinamento e correspondem gravado. Os resultados indicam que os jogadores antes da lesáo manifesta níveis mais elevados de estresse e auto-confiança, e depois de sofrer uma lesão que se manifesta níveis mais elevados de depressão, ansiedade cognitiva e ansiedade somática. Os resultados sâo discutidos e aplicaçôes práticas deles derivados são propostas.

Palavras chave: Estado de humor, ansiedade pré-concorrencial, lesóes do esporte, jogadores de futebol.

\section{Introducción}

La lesión es uno de los hechos cotidianos de la práctica deportiva con el que deben de convivir los deportistas y los pro-

Dirección para correspondencia [Correspodence address]: Aurelio Olmedilla Zafra. Universidad de Murcia. Facultad de Psicología Departamento de Personalidad, Evaluación y Tratamiento Psicológico. Campus Universitario de Espinardo. 30800 Murcia - España. E-mail: olmedilla@um.es fesionales del deporte. Si en todos los deportes se producen lesiones, en el fútbol se da una elevada incidencia, en torno a 9 lesiones por 1000 horas de juego, sea en entrenamientos o partidos (Cos, Cos, Buenaventura, Pruna y Ekstrand, 2010; Ekstrand, 2008; Ekstrand, Waldén y Hägglund, 2002; Hägglund y Waldén, 2012; Noya y Sillero, 2012). Los esfuerzos de los investigadores por estudiar con precisión las 
lesiones en el fútbol han posibilitado cierto consenso en la evaluación de las lesiones (Dvorak, Junge, Derman y Schwellnus, 2011; Fuller et al., 2006; Junge et al., 2004) y en la consideración de aproximaciones multidisciplinares para su prevención y rehabilitación (Paredes et al., 2012; Olmedilla y García-Mas, 2009; Wiese-Bjornstal, 2010).

Uno de los aspectos que en los últimos años ha sido considerado en la etiología de las lesiones y en los procesos de rehabilitación de las mismas ha sido el factor psicológico. Las relaciones entre lesión y aspectos psicológicos del deportista están bien documentadas (Ardern, Taylor, Feller y Webster, 2013; Junge, 2000; Wiese-Bjornstal, 2010), y la influencia recíproca entre ellas se van constatando cada día, si bien todavía quedan muchos interrogantes por responder (Olmedilla y García-Mas, 2009). La literatura científica indica que el riesgo, la causalidad y la etiología de la lesión deportiva incluyen la interacción de aspectos intrínsecos (características biológicas y psicológicas) y de aspectos extrínsecos (características físicas y socio-culturales) y su influencia en la conducta del deportista (Wiese-Bjornstal, 2009); en este sentido algunos autores realzan la importancia que tienen los procesos cognitivos, emocionales y conductuales tanto en la ocurrencia de lesiones como en la rehabilitación de las mismas (Hackfort y Kleinert, 2007; Heniff, 1998; Wiese-Bjornstal, Smith, Shaffer y Morrey, 1998).

Las teorías que han intentado explicar estas relaciones provienen de modelos psicológicos específicos (Andersen y Williams, 1988; Brewer, 1994; Heil, 1993; Pedrosa, SotoSánchez, Suárez-Álvarez y García-Cueto, 2012), de modelos biomédicos (Meeuwisse, Tyreman, Hagel y Emery, 2007) y de modelos biopsicosociales (Wiese-Bjornstal, 2009; WieseBjornstal et al., 1998).

Las aportaciones del modelo de Andersen y Williams (1988) revalorizaron el papel del estrés en la aparición de lesiones deportivas, y su revisión posterior (Williams y Andersen, 1998) constató la respuesta de estrés como el resultado de una relación bidireccional entre las valoraciones cognitivas del deportista sobre una situación externa, potencialmente estresante, y los aspectos fisiológicos y atencionales del estrés, donde tanto estas valoraciones cognitivas como las respuestas fisiológicas y atencionales ante el estrés, se modifican constantemente. En esta línea el modelo de Wiese-Bjornstal et al. (1998) señala que si bien determinados aspectos psicológicos, con el estrés como central, influyen en la aparición de lesiones, éstas a su vez influyen en las reacciones psicológicas y emocionales del deportista.

En esta relación bidireccional entre psicología-lesión-psicología algunas de las variables a considerar por su relevancia en el ámbito competitivo son la ansiedad y el estado de ánimo del deportista. Sin embargo las dificultades de evaluación de la ansiedad precompetitiva, la manifestada justo antes de iniciar la competición, y el estado de ánimo previo a la mis- ma han impedido una mayor investigación, sobre todo con deportistas profesionales o semiprofesionales. En el caso de la ansiedad se han realizado, en la mayoría de los casos, estudios de la ansiedad rasgo con diseños descriptivos y transversales (Aslan, Aslan y Alparslan, 2000; Kleinert, 2002; Olmedilla, Andreu, Ortín y Blas, 2010; Petrie, 1993; Ye, 2002), y en el caso de los estados de ánimo se han realizado fundamentalmente con deportistas en proceso de rehabilitación (Dessoki, El-Kalupy y Hefnawy, 2012; Rozen y Horne, 2007).

Por todo ello, y siguiendo las sugerencias metodológicas en la investigación de los aspectos psicológicos de las lesiones deportivas, realizadas por diferentes autores (Olmedilla y García-Mas, 2009; Petrie y Falkstein, 1998; Wiese-Bjornstal, 2010) se ha llevado a cabo el presente estudio, con un diseño longitudinal, con una muestra homogénea y en el contexto de su práctica deportiva real, en este caso la competición.

Así, el objetivo del presente estudio es analizar la incidencia de la lesión sobre los posibles cambios en el estado de ánimo y en la ansiedad precompetitiva de los futbolistas.

\section{Método}

\section{Participantes}

La muestra estuvo formada por 13 jugadores de equipos de fútbol masculino del Grupo XIII de la Tercera División Nacional de la Federación de Fútbol de la Región de Murcia, que se lesionaron durante el desarrollo de la temporada 2011-2012. El rango de edad fue de 21 a 29 ańos, con una media de 24.58 años (DT=3.42). Del total de jugadores dos eran porteros, cuatro defensas, cuatro medios y tres delanteros. Respecto a la gravedad de las lesiones todos los jugadores analizados tuvieron una lesión de tipo moderada (Olmedilla, García-Montalvo y Martínez-Sánchez, 2006).

\section{Diseño}

Se llevó a cabo un diseño longitudinal, en el se tomaron los datos durante una temporada completa de competición, evaluando en cada partido oficial de competición la ansiedad competitiva, el estado de ánimo, y se registraron las lesiones producidas en entrenamiento y en partido.

\section{Procedimiento}

En el proceso de la investigación pueden considerarse 4 fases. En primer lugar una toma de contacto con el club, de cara a la petición de los permisos pertinentes y explicación de la investigación. En segundo lugar, se realizó una entrevista con el entrenador y el director deportivo del club al objeto de solicitar la colaboración en el registro de lesiones, así como la explicación de la hoja de registro a utilizar. En un tercer 
momento, se llevó a cabo la cumplimentación de los cuestionarios por parte de los futbolistas, se realizó antes de cada partido (aproximadamente una hora y media antes, y no llevó más de 5 minutos su cumplimentación) con el fin de ser lo más próximo a la actividad, pero sin afectar al inicio de sus actividades de calentamiento. Por último y en cuarto lugar, los registros de las lesiones se llevaron a cabo durante toda la temporada, semana por semana, por parte del fisioterapeuta, que fue el encargado del seguimiento de las lesiones.

\section{Instrumentos}

Para evaluar la ansiedad precompetitiva se utilizó el Inventario de Ansiedad Competitiva, Competitive State Anxiety Inventory-2 (CSAI-2) de Martens, Burton, Vealey, Bump y Smith (1990) en la versión española de Márquez (1992). Está formado por 27 ítems, de opciones de respuesta tipo Lickert; a cada afirmación la persona debe responder seleccionando la opción que refleje su estado competitivo ( $1=\mathrm{N} a d a ; 2=U n$ poco 3 = Moderadamente $;$ = Mucho). Los ítems se encuentran distribuidos en tres subescalas que miden ansiedad cognitiva, ansiedad somática y autoconfianza. El coeficiente alfa de Cronbach en la versión original mostró una consistencia interna entre .79 y .90 , y en la versión española entre .51 y .86 .

Para la evaluación de los estados de ánimo se ha utilizado el Profile of Mood States (POMS) de McNair, Lorr y Droppleman (1971), en la versión española abreviada de 29 ítems de Fuentes, Balaguer, Meliá y García-Merita (1995). Esta versión del POMS incluye una escala tipo Likert con valores de 0 (nada) a 4 (muchísimo) para evaluar cinco dimensiones: tensión, depresión, cólera, vigor y fatiga. Este instrumento de medida es el más utilizado para evaluar esta variable en el ámbito deportivo y el más citado en la literatura científica (Andrade, Arce y Seoane, 2000), y aunque necesitado de mayor investigación con muestras españolas (Andrade, Arce y Seoane, 2002; Arce, Andrade y Seoane, 2000), ha demostrado su utilidad como medida del estado de ánimo así como su relación con el rendimiento deportivo (De la Vega, Ruiz, García-Mas, Balagué, Olmedilla y Del Valle, 2008), las lesiones deportivas y su periodo de recuperación (Alzate, Ramírez y Artaza, 2004; Ramírez, Alzate y Lázaro, 2002).

Para la evaluación y seguimiento de las lesiones deportivas se utilizó una hoja de registro específica (Olmedilla, Laguna y Blas, 2011, adaptada de Díaz, 2001) que cumplimentaban los fisioterapeutas.

\section{Análisis estadístico}

Para el análisis de los datos, y con el objeto de conocer si existían diferencias entre las medias de los partidos antes de lesionarse con las medias de los partidos después de lesionarse en las variables psicológicas, se utilizó la prueba no paramétrica para la comparación de medias con 2 muestras relacionadas de Wilconxon, utilizando un nivel de significación de $\mathrm{p}<.05$.

\section{Resultados}

En la Tabla 1 se indican los valores medios de cada una de las variables, objeto de estudio, registradas desde el comienzo de la temporada hasta la misma semana de la lesión, y desde la semana de su incorporación hasta final de temporada.

Tabla 1. Valores de ansiedad competitiva y estados de ánimo antes de la lesión, y después de la lesión

\begin{tabular}{llll}
\hline Variable & Antes de la lesión & Después de la Lesión & Z Valor \\
\hline Tensión & 5,432 & 3,7945 & .026 \\
Depresión &, 38 & 1,187 & .075 \\
Cólera & 5,346 & 4,6008 & .382 \\
Vigor & 11,500 & 11,6354 & .650 \\
Fatiga &, 577 &, 9854 & .440 \\
Autoconfianza & 30,833 & 29,2517 & .071 \\
Ansiedad cognitiva & 18,625 & 23,1125 & .004 \\
Ansiedad somática & 12,021 & 14,1467 & .060 \\
\hline
\end{tabular}

Los datos de la Tabla 1 señalan que los valores de Tensión, Cólera y Autoconfianza eran mayores antes de la lesión que después, apreciándose diferencias estadísticamente significativas en Tensión, tendencias a la significación en Autoconfianza, y no se apreciaron diferencias en Cólera.

Por el contrario se apreciaron valores superiores tras recibir la lesión en las variables Depresión, Vigor, Fatiga, Ansiedad cognitiva y Ansiedad somática, de manera que se registraron diferencias estadísticamente significativas en Ansiedad cognitiva, tendencias a la significación en Ansiedad somática, Depresión, y no se apreciaron diferencias en Vigor ni en Fatiga. 


\section{Discusión}

El objetivo del presente estudio ha sido analizar la incidencia de la lesión sobre los posibles cambios en el estado de ánimo y en la ansiedad precompetitiva de los futbolistas. La literatura científica indica que tanto el estado de ánimo como la ansiedad precompetitiva se asocian al rendimiento deportivo (De la Vega, Ruiz, García y del Valle, 2011; Terry, 1997) por lo que la afectación de las lesiones a estos estados psicológicos puede mermar el rendimiento de los jugadores, no solo por la pérdida de entrenamientos y partidos de competición, sino por una disposición psicológica no adecuada. Los resultados más evidentes del presente estudio indican que los futbolistas manifestaban antes de la lesión niveles superiores de Tensión y de Autoconfianza; y después de haber sufrido una lesión manifestaban niveles superiores de Depresión, Ansiedad cognitiva y Ansiedad somática.

Se puede afirmar entonces que los resultados muestran que efectivamente existe una influencia de la lesión en el estado de ánimo y en la ansiedad precompetitiva. Respecto al estado de ánimo los futbolistas mostraron unos niveles superiores de Tensión (estadísticamente significativos) y de Cólera (sin significación estadística) antes de la lesión; y por el contrario los futbolistas mostraron niveles superiores de Depresión, Vigor y Fatiga después de la lesión, aunque solo en Depresión tendían a la significación estadística. En cualquiera de los dos casos alejados del perfil de iceberg propuesto por Morgan (1980) donde el factor Vigor debería estar por encima de la media del resto de factores. Se puede indicar, por tanto, que las lesiones afectan al estado de ánimo, y lo hacen negativamente provocando cambios que, en algunos aspectos llegan a ser significativos, y que suelen ser muy importantes al limitar la competencia deportiva y el rendimiento derivado de la misma. Por otro lado, los estados elevados de tensión se relacionan con el riesgo a sufrir lesión (Andersen y Williams, 1988; Díaz, Buceta y Bueno, 2004; Williams y Andersen, 1998), y la depresión como un estado anímico no adecuado ni para la recuperación de la lesión (Abenza, Olmedilla, Ortega, Ato y García-Mas, 2010; Appaneal, Levine, Perna y Roth, 2009; Dessoki, El-Kalupy y Hefnawy, 2012; Ramírez et al., 2002), ni para el rendimiento deportivo (De la Vega et al., 2008; De la Vega et al., 2011).

Rozen y Horne (2007), con deportistas que practicaban fútbol australiano, evaluaron el estado de ánimo y la ansiedad de éstos en la pretemporada y cuando sufrían una lesión, indicando que el factor Vigor era un buen predictor de lesión durante el transcurso de la temporada, en el sentido de que altas puntuaciones en este factor predecían una baja probabilidad de sufrir lesión y que bajas y medias puntuaciones predecían una alta probabilidad de sufrir lesión, lo que ratifica el perfil de iceberg de Morgan (1980), y lo hace no solo como predicción de buen rendimiento (De la Vega et al., 2008; Morgan y Pollock, 1977), sino como indicador de protección ante la lesión. Además, cuando se producía una lesión la afectación al estado de ánimo era evidente, y sobre todo cuando ésta era de larga duración, produciéndose un descenso de Vigor, un incremento de Fatiga, Confusión, Tensión e Ira y niveles altos de Depresión, lo que está en línea con lo hallado en nuestro estudio. La explicación que ofrecen Rozen y Horne (2007) respecto a la influencia del estado de ánimo en la ocurrencia de lesiones se sitúa en la línea del modelo de salud mental de Morgan (1980) con el perfil de iceberg, es decir, que los futbolistas con puntuaciones altas en Vigor se adecúan mejor a los requerimientos del fútbol, a su intensidad y a aquellas situaciones con un potencial elevado de riesgo a la lesión (encontronazos, entradas de adversarios, etc.), lo que puede permitirle adaptarse mejor y ser más competente en prevenir la lesión. Y todo lo contrario ocurre con los futbolistas con bajos niveles de Vigor.

Respecto a la ansiedad precompetitiva, los futbolistas del presente estudio mostraron niveles superiores de Autoconfianza antes de la lesión, con tendencia a la significación estadística, datos en línea con lo encontrado por Olmedilla et al. (2006) también con una muestra de futbolistas y que corroboran lo hallado por Petrie (1993) en su estudio, indicando que los niveles altos de autoconfianza podrían influir en la conducta del deportista, asumiendo más riesgos, y en su capacidad atencional, reduciendo un estado de alerta adecuado al contexto, y por tanto en ambos casos, incrementando la probabilidad de lesionarse. En este sentido, algunos estudios han encontrado un factor de competencia percibida junto a otros como la búsqueda de sensaciones, la asunción de riesgos, la percepción del riesgo y la competitividad para evaluar la predisposición del deportista a sufrir lesiones o accidentes deportivos (Latorre y Pantoja, 2013).

Sin embargo, tras recibir una lesión, los futbolistas del presente estudio mostraron niveles superiores de Ansiedad cognitiva, en línea con lo encontrado por Rozen y Horne (2007), lo que parece indicar un aumento de los pensamientos negativos y de la preocupación, aspectos que sumados a los niveles superiores también de Ansiedad somática, merman la competencia deportiva del jugador, restándole capacidad de rendimiento, y aumentando a su vez la probabilidad de sufrir lesión (Ivarsson, Johnson y Podlog, 2013; Olmedilla et al., 2006; Olmedilla et al., 2010; Petrie, 1993), además de limitar su proceso de rehabilitación, donde la depresión y la ansiedad juegan un papel relevante (Dessoki et al., 2012), sobre todo en aquellas lesiones graves, como por ejemplo las de ligamento cruzado anterior, donde el factor psicológico es primordial para volver al nivel previo a la lesión (Ardern et al., 2013; Brewer et al., 2007).

Los resultados de este estudio abogan, en la línea de lo apuntado también por Rozen y Horne (2007), por la necesidad de una mayor investigación sobre los efectos del estado 
de ánimo en la probabilidad de sufrir lesión, por un lado, y sobre los efectos de la lesión deportiva en el estado de ánimo del deportista. Las pocas investigaciones realizadas con diseños como el llevado a cabo en este trabajo indican tendencias muy interesantes respecto a que factores psicológicos juegan un papel importante tanto en la incidencia como en la recuperación de lesiones. Y más cuando se analiza con una de las variables más relevantes en la investigación de las lesiones como la ansiedad precompetitiva, y que en pocas ocasiones se ha estudiado longitudinal y ecológicamente. La asociación del factor Vigor y del factor Depresión en el estudio de Rozen y Horne (2007) y del factor Tensión, y de Autoconfianza y Ansiedad cognitiva en nuestro estudio con la incidencia y recuperación de lesiones resultan esperanzadores para continuar este tipo de investigaciones. Además, se deberían de continuar con diseños de tipo prospectivo y longitudinal, quizá con un mayor número de sujetos, y también con un mayor periodo de investigación, lo que ayudaría a establecer mejor el papel que juega el estado de ánimo y la ansiedad precompetitiva en la etiología de la lesión, así como en las recidivas, aspecto que ha sido muy poco estudiado por los investigadores.

\section{Aplicaciones prácticas}

Estos resultados podrían tener implicaciones en la prevención y en el tratamiento de la lesión deportiva. Se puede considerar que determinados programas de entrenamiento psicológico ayudarían a los deportistas a afrontar mejor las situaciones deportivas en las que se encuentran. Sería muy importante, y se debería recomendar a entrenadores y gestores de clubes y equipos deportivos, poder trabajar psicológicamente con los futbolistas mediante programas específicos de control de estrés y control emocional, lo que podría incrementar su fortaleza mental, aspecto que puede permitir afrontar mejor el estrés que implica la práctica deportiva y por lo tanto, tal y como indican Petrie, Deiters y Harmison (2013) minimizar la vulnerabilidad a sufrir lesión, y en caso de lesión afrontar ésta con respuestas psicológicas y emocionales adaptativas mejorando las estrategias en el periodo de rehabilitación (Wadey, Evans, Hanton y Neil, 2012).

Desde una perspectiva de prevención, Edvardsson, Ivarsson y Johnson (2012) resaltan las investigaciones que han mostrado la reducción de lesiones deportivas a través del entrenamiento en habilidades psicológicas, sobre todo con programas cognitivo-conductuales para el control del estrés, utilizando técnicas como la visualización y la relajación, quizá las más usadas en este tipo de intervenciones (Olmedilla, Ortega, Abenza y Boladeras, 2011), y en línea con el planteamiento del modelo de Pedrosa et al. (2012), alternativo al de Andersen y Williams (1988), y en el que se confirma el papel relevante de las habilidades de control del estrés como variable mediadora respecto a las lesiones deportivas.

Además, un ámbito cada día más necesitado de intervención psicológica es el momento de la vuelta a la práctica deportiva tras una lesión grave, donde aparecen problemas de ansiedad e inseguridad por parte del deportista, generalmente relacionados con la percepción de competencia; para Podlog, Dimmock y Miller (2011) las estrategias de intervención psicológica basadas en la teoría de la autodeterminación pueden dar muy buen resultado cuando son dirigidas a los profesionales de la medicina deportiva, médicos, fisioterapeutas, recuperadores, etc.

\section{Referencias}

1. Abenza, L., Olmedilla, A., Ortega, E., Ato, M. y García-Mas, A. (2010). Análisis de la relación entre el estado anímico y las conductas de adherencia en deportistas lesionados. Anales de Psicología, 26(1), 159-168.

2. Alzate, R., Ramírez, A. y Artaza, J. L. (2004). The Effect of Psychological Response on Recovery of Sport Injury. Research in Sports Medicine, $15,15-31$.

3. Andersen, M.B. y Williams, J.M. (1988). A model of stress and athletic injury: Prediction and prevention. Journal of Sport and Exercise Psychology, 10, 294-306

4. Andrade, E. M., Arce, C. y Seoane, G. (2000). Aportaciones del POMS a la medida del Estado de Ánimo de los deportistas: estado de la cuestión. Revista de Psicología del Deporte, 9(1-2), 7-20.

5. Andrade, E. M., Arce, C. y Seoane, G. (2002). Adaptación al español del cuestionario "Perfil de los Estados de Ánimo" en una muestra de de-portistas. Psicothema, 14(4), 708-713.

6. Appaneal, R.N., Levine, B.R., Perna, F.M. y Roh, J.L. (2009). Measuring post-injury depression among male and female competitive athletes. Journal of Sport and Exercise Psychology, 31, 60-76.

7. Arce, C., Andrade, E. M. y Seoane, G. (2000). Problemas semánticos en la adaptación del POMS al castellano. Psicothema, 12(2), 47-51.

8. Ardern, C.L., Taylor, N.F., Feller, J.A., Whitehead, T.S. y Webster, K.E. (2013). Psychological responses matter in returning to preinjury level of sport after anterior cruciate ligament reconstruction surgery. American Journal of Sports Medicine, 41(7), 1549-1558.

9. Aslan, S.H., Aslan, R.O. y Alparslan, Z.N. (2000). Anxiety levels of the football players participating in the U-21 national team infrastructure selection. Turkish Journal of Sports Medicine, 35(2), 51-58.

10. Brewer, B.W. (1994). Review and critique of models of psychological adjustment to athletic injury. Journal of Applied Sport Psychology, 6, 87100.

11. Brewer, B.W., Cornelius, A.E., Sklar, J.H., Van Raalte, J.L., Tennen, H., Armeli, S., Corsetti, J.R. y Brickner, J.C. (2007). Pain and negative mood during rehabilitation after anterior cruciate ligament reconstruction: a daily process analysis. Scandinavian Journal of Medicine and Sciences in Sport, 17, 520-529.

12. Cos, F., Cos, M.A., Buenaventura, L. Pruna, R. y Ekstrand, J. (2010). Modelos de análisis para la prevención de lesiones en el deporte. Estudio epidemiológico de lesiones: el modelo Union of European Football Associations en el fútbol. Apunts, Medicina de l'Esport, 45(166), 95-102.

13. De la Vega, R., Ruiz, R., García-Mas, A., Balagué, G., Olmedilla, A. y Del Valle, S. (2008). Consistencia y fluctuación de los estados de ánimo en un equipo de fútbol profesional durante una competición de play off. Revista de Psicología del Deporte, 17(2), 241-251.

14. De la Vega, R., Ruiz, R., García, G.D. y del Valle, S. (2011). El estado de 
ánimo precomnpetitivo en un equipo de fútbol profesional: un estudio entre jugadores titulares y suplentes. Cuadernos de Psicología del Deporte, 11(2), 107-117.

15. Dessoki, H., El-Kalupy, H. y Hefnawy, T. (2012). Psychological effect of lower limb injuries among football players. 20th European Congress of Psychiatry.

16. Díaz, M.P., Buceta, J.M. y Bueno, A.M. (2004). Situaciones estresantes y vulnerabilidad a las lesiones deportivas: un estudio con deportistas de equipo. Revista de Psicología del Deporte, 14(1), 7-24.

17. Díaz, P. (2001). Estrés y prevención de lesiones. Tesis doctoral no publicada. Universidad Nacional de Educación a Distancia.

18. Dvorak, J., Junge, A., Derman, W. y Schwellnus, M. (2011). Injuries and illnesses of football players during the 2010 FIFA World Cup. British Journal of Sports Medicine, 45(8), 626-630.

19. Edvardsson, A., Ivarsson, A. y Johnson, U. (2012). Is a cognitive-behavioural biofeedback intervention useful to reduce injury risk in junior football players?. Journal of Sports Science and Medicine, 11, 331-338.

20. Ekstrand, E. (2008). Epidemiology of football injuries. Science and Sports, 23, 73-77.

21. Ekstrand, E., Waldén, M. y Hägglund, M. (2002). A congested football calendar and the wellbieng of players: Correlation between macht exposure of European footballers before the World Cup Epidemiology of football injuries. Science and Sports, 23, 73-77.

22. Fuentes, I., Balaguer, I., Meliá, J. L. y García-Merita, M. L. (1995). Forma abreviada del Perfil de los Estados de Ánimo (POMS). Actas del $V$ Congreso Nacional de Psicología de la Actividad Física y el Deporte, (pp. 29-39). Valencia. Universitat de València.

23. Fuller, C.W., Ekstrand, J., Junge, A., Andersen, T.E., Bahr, R., Dvorak, J., Hágglund, M., McCrory, P. y Meeuwisse, W.H. (2006). Consensus statement on injury definitions and data collection procedures in studies of football (soccer) injuries. British Journal of Sports Medicine, 40, 193-201.

24. Hackfort, D. y Kleinert, J. (2007). Research on Sport Injury Development: Former and Future Approaches from an Action Theory Perspective. International Journal of Sport Exercise and Psychology, 5, 324-339.

25. Hägglund, M. y Waldén, M. (2012). Epidemiology of football injuries. Dansk SportsMedicin, 4(16), 10-12.

26. Heil, J. (1993). Psychology of sport injury. Champaign, Il: Human Kinetics.

27. Heniff, C.B. (1998). A comparison of life event stress, weekly hassles, and mood disturbance between injured and uninjured female university athletes. Unpublished masters' thesis, University of Minnesota, Available TC Wilson Library Theses.

28. Ivarsson, A., Johnson, U. y Podlog, L. (2013). Psychological predictors of injury ocurrence: A prospective investigation of professional swedish soccer players. Journal of Sport Rehabilitation, 22(1), 19-26.

29. Junge, A. (2000). The influence of psychological factors on sports injuries: Review of the literature. American Journal of Sports Medicine, 28, $10-15$

30. Junge, A., Langevoort, G., Pipe, A., Peytavin, A., Wong, F., Mountjoy, M., Beltrami, G., Terrell, R., Holzgraefe, M., Charles, R. y Dvorak, J. (2004). Injuries in team sport tournaments during the Olympic games. American Journal of Sports Medicine, 34, 565-576.

31. Kleinert, J. (2002). An approach to sport injury trait anxiety: scale construction and structure analysis. European Journal of Sport Science, 2(3), 49-57.

32. Latorre, P.A. y Pantoja, A. (2013). Diseńo y validación de un cuestionario de propensión al accidente deportivo. Cuadernos de Psicología del Deporte, 13(1), 51-62.

33. Márquez, S. (1992). Instrumentos de Evaluación de la Ansiedad: Aplicación al Ámbito de la Competición Deportiva. Perspectivas de la Actividad Fisica y el Deporte, 9, 13-17.

34. Martens, R., Burton, D., Vealey, R.S., Bump, L.A. y Smith, P.E. (1990).
Development and validation of the competitive state Anxiety Inventory-2. En R. Martens, R.S. Vealey y D. Burton (eds.), Competitive Anxiety in Sport, (pp. 117-190). Champaigne, IL: Human Kinetics.

35. McNair, D. M., Lorr, M., y Droppleman, L. F. (1971). Profile of Mood States Manual. San Diego, CA: Educational and Industrial Testing Services.

36. Meeuwisse, W.H., Tyreman, H., Hagel, B. y Emery, C. (2007). A dynamic model of etiology in sport injury: the recursive nature of risk and causation. Clinical Journal of Sports Medicine, 17(3), 215-219.

37. Morgan, W.P. (1980). Test of champions: The iceberg profile. Psychology Today, 14, 92-99.

38. Morgan, W.P. y Pollock, M. (1977). Psychological characterization of the elite distance runner. Annals of the New York Academy of Science, 301, 382-403.

39. Noya, J. y Sillero, M. (2012). Epidemiología de las lesiones en el fútbol profesional español en la temporada 2008-2009. Archivos de Medicina del Deporte, XXIX(150), 750-766.

40. Olmedilla, A. y García-Mas, A. (2009). El Modelo Global Psicológico de las Lesiones Deportivas. Acción Psicológica, 6(2), 77-91.

41. Olmedilla, A., Andreu, M.D., Ortín, F.J. y Blas, A. (2010). Competitive anxiety and injuries: Sports factors, types and injury gravity. International Journal of Hispanic Psychology, 3(2), 34-46.

42. Olmedilla, A., García-Montalvo, C. y Martínez-Sánchez, F. (2006). Factores psicológicos y vulnerabilidad a las lesiones deportivas: un estudio en futbolistas. Revista de Psicología del Deporte, 15(1), 37-52.

43. Olmedilla, A., Laguna, M. y Blas, A. (2011). Lesiones y características psicológicas en jugadores de balonmano. Revista Andaluza de Medicina del Deporte, 4(1), 6-12.

44. Olmedilla, A., Ortega, E., Abenza, L. y Boladeras, A. (2011). Lesiones deportivas y psicología: una revisión (2000-2009). Cuadernos de Psicología del Deporte, 11(1), 45-57.

45. Paredes, V., Gallardo, J., Porcel, D., de la Vega, R., Olmedilla, A. y Lalín, C. (2012). La readaptación físico-deportiva de lesiones. Aplicación práctica metodológica. Madrid: OnXsport.

46. Pedrosa, I., Soto-Sánchez, A., Suárez-Álvarez, J. y García-Cueto, E. (2012). Adaptación española de una Escala de Apoyo Social percibido para deportistas. Estudios de Psicología, 33(3), 359-369.

47. Petrie, T.A. (1993). Coping skills, competitive trait anxiety, and playing status: Moderating effects on the life stress-injury relationship. Journal of Sport and Exercise Psychology, 15, 261-274.

48. Petrie, T.A. y Falkstein, D.L. (1998). Methodological, measurement and statistical issues in research on sport injury prediction. Journal of Applied Sport Psychology, 10, 26-45.

49. Petrie, T.A., Deiters, J. y Harmison, R.J. (2013). Mental toughness, social support, and athletic identity: Moderators of the life stress-injury relationship in collegiate football players. Sport, Exercise, and Performance Psychology, doi: 10.1037/a0032698.

50. Podlog, L., Dimmock, J. y Miller, J. (2011). A review of return to sport concerns following injury rehabilitation: Practitioner strategies for enhancing recovery outcomes. Physical Therapy in Sport, 12(1), 36-42.

51. Ramírez, A., Alzate, R. y Lázaro, I. (2002). Efectos psicológicos de la lesión deportiva. Motricidad, 9, 209-224.

52. Rozen, W.M. y Horne, D.J.L. (2007). The Association of Psychological Factors with Injury. Incidence and Outcome in the Australian Football League. Individual Differences, 5(1), 73-80.

53. Terry, P.C. (1997). The application of mood profiling with elite sport performers. En R.J. Butler (Ed.), Sport Psychology in performance, (pp. 3-32). New York: Plenum Press.

54. Wadey, R., Evans, L., Hanton, S. y Neil, R. (2012). An examination of hardiness throughout the sport injury process. British Journal of Health Psychology, 17, 103-128.

55. Wiese-Bjornstal, D.M. (2009). Sport injury and college athlete health across the lifespan. Journal of Intercollegiate Sport, 2, 64-80. 
56. Wiese-Bjornstal, D.M. (2010). Psychology and socioculture affect injury risk, response, and recovery in high-intensity athletes: a consensus statement. Scandinavian Journal of Medicine and Sciences in Sport, 20(Supp. 2), 103-111.

57. Wiese-Bjornstal, D.M., Smith, A.M., Shaffer, S.M. y Morrey, M.A. (1998). An integrated model of response to sport injury: Psychological and sociological dynamics. Journal of Applied Sport Psychology, 10, 4669.
58. Williams, J.M. y Andersen, M.B. (1998). Psychological Antecedents of Sport Injury: Review and Critique of the Stress and Injury Model. Journal of Applied Sport Psychology, 10, 5-25.

59. Ye, P. (2002). Differences of competitive trait anxiety between athletes. Journal of Beijing University of Physical Education, 25(2), 183-185. 